\title{
Effect of the Treatment with Alpha-Macroglobulin on the Development of Experimental Pancreatitis
}

\author{
Authors \\ V. E. Di Loreto, S. M. Roma, A. Rigalli \\ Affiliation \\ Bone Biology Laboratory, School of Medicine Rosario National University, Rosario, Argentina
}

Key words
macroglobulin
monofluorophosphate
MFP
pancreas

received 01.08 .2012 accepted 11.12 .2012

Bibliography DOI http://dx.doi.org/ 10.1055/s-0032-1333227 Published online: January 17, 2013 Drug Res 2013; 63: 90-93 (c) Georg Thieme Verlag KG Stuttgart · New York ISSN 2194-9379

Correspondence

\section{A. Rigalli}

Laboratorio de Biología Ósea

Facultad de Medicina

Santa Fe 3100

2000 Rosario

Argentina

Tel.: +54/341/15578 6840

Fax: $+54 / 341 / 4804569$

arigalli@conicet.gov.ar

\section{Abstract \\ $\nabla$}

Alpha-macroglobulins are proteinase inhibitors. Monofluorophosphate increases alpha-macroglobulin levels in plasma, inducing a higher survival rate and lower pancreatic damage in rats with pancreatitis. The aim of this study was to evaluate the effect of alpha-macroglobulin on the development of pancreatitis. Pancreatitis was surgically induced in Sprague-Dawley rats divided into groups of 16 rats each and subjected to the following intravenous treatments for 3 days: Controls: pancreatitis without treatment, Enriched plasma: pancreatitis + alpha-macroglobulin-enriched plasma, Normal

\section{Introduction}

$\nabla$

Acute pancreatitis is an inflammatory disease of the pancreas characterized by auto-digestion of the gland as a consequence of a release and premature activation of pancreatic enzymes [1]. The development of pancreatitis depends partially on the balance between proteinases and their inhibitors [2]; the pathogenic mechanisms involved in the development of the disease are not well understood. Alpha-macroglobulins (AM) are a family of plasma proteins whose main function is the inactivation of proteinases [3]. The binding of AM to trypsin determines the fragmentation and clearance of the AM-trypsin complex [4]. The fragmentation process is detected in human pancreatitis and correlates with the degree of progression to multiple organ failure [5].

Studies using antiproteolytic agents in animal models have shown an improvement in the course of the disease. But whether this is effective for treatment of acute pancreatitis in humans remains unclear [6]. Several studies are being conducted where anti-citokine therapy is used as damage control strategy in order to modulate the inflammatory response and so, mitigate systemic effects and organ failure [7]. plasma: pancreatitis + plasma with normal levels of alpha-macroglobulin, Saline Solution: pancreatitis + saline solution, Purified alpha-macroglobulin: pancreatitis+ purified alpha-macroglobulin. After 14 days pancreatic damage was assessed using a score that measures: edema, fibrin, neutrophils, mononuclear leukocytes, necrosis, vascular congestion, thrombosis, hemorrhage and fibrosis. Pancreatic damage decreased and the percentage of animals with pancreatitis was lower in enrichedplasma and purified alpha-macroglobulin groups. We conclude that the intravenous administration of alpha-macroglobulins causes a reduction in the histological damage produced by pancreatitis.
Sodium monofluorophosphate (MFP) is a drug used for the treatment of osteoporosis [8] that binds to plasma AM [9]. It has been demonstrated that the administration of MFP for 30 days increases plasma levels of AM in rats $[10,11]$ and modifies the course of pancreatitis [11]. The administration of MFP before the induction of pancreatitis showed a protective effect on the pancreatic parenchyma. Morover, those animals that received MFP after the surgical induction of acute pancreatitis showed less pancreatic damage than rats that did not receive MFP [12]. Animals pre-treated with MFP showed an increase in the concentration of AM, so it could be assumed that the protective role of the drug could be due to a change in the homeostasis of AM that favors the inactivation of the proteinases. There is a direct association between the reduced pancreatic injury, the greater survival rate of MFP treated animals and the increase in plasma AM levels. However, there is no strong evidence that this change in the homeostasis of AM is the cause of the observed effects.

The present study was designed to assess the effect of AM on the development of experimental pancreatitis in rats. 


\section{Materials and Methods}

$\nabla$

The study was carried out on 7-week-old male Sprague-Dawley rats. All experiments were performed in accordance with the international ethical guidelines for animal care [13]. Acute pancreatitis was induced by incomplete closed duodenal loop (ICDL) $[12,14]$ in 80 rats.

After induction of pancreatitis, the animals were randomly divided into groups of 16 animals each. Information about pain was analyzed and rats euthanized when pain and suffering were excessive. Amylase, AM concentration and the histological damage score shown in this work belong to those animals that survived 14 days after induction of pancreatitis; for this reason the number of rats initially assigned to each group $(n=16)$ is not the same as that indicated below for each group.

1) Controls $(C, n=10)$ : rats received no treatment after ICDL.

2) Pancreatitis $+\mathrm{NaCl} 0.9 \%(\mathrm{SS}, \mathrm{n}=14)$ : Rats received an intravenous (iv) injection of $0.5 \mathrm{ml}$ of saline solution in the tail vein daily for $72 \mathrm{~h}$ after ICDL [15].

3) Pancreatitis + normal AM plasma levels (ANP, $n=14$ ): Rats received an iv injection of $0.5 \mathrm{ml}$ of plasma with normal AM levels daily for $72 \mathrm{~h}$ after ICDL.

4) Pancreatitis + AM enriched plasma (AEP, $n=16)$ : Rats received daily for $72 \mathrm{~h}$ after ICDL an iv injection of $0.5 \mathrm{ml}$ of plasma with levels higher than normal of AM.

5) Pancreatitis + purified AM (PAM, n=14): Rats received an iv injection of $0.5 \mathrm{ml}$ with purified AM daily for $72 \mathrm{~h}$ after ICDL.

6) A group of 16 rats underwent the same experimental conditions described before but with only a median laparotomy and suture of the abdominal wall, without ICDL procedure (Sham group).

Blood samples were drawn in order to measure plasma amylase activity (Wiener Lab, Rosario, Argentina) and AM levels [11].

Animals were euthanized 14 days after surgery [16]. Pancreatic and peri-pancreatic adipose tissues were removed. Microscopic observations were performed by one experienced researcher masked to the specific experimental condition of each preparation (SMR).

\section{Histo-pathological Score}

Pancreatic damage was evaluated using a numerical scoring system [17]. Interstitial edema, fibrin deposition, infiltration of neutrophils and mononuclear cells, fatty tissue and parenchymal necrosis, congestive blood vessels, hemorrhage, vascular thrombosis and fibrosis were all assessed. These parameters were considered markers of injury [18] and they were graded independently with a qualitative scale. Number 0 was assigned if there were no changes, 1 to minimum changes, 2 to moderate, 3 to severe. The final score for each rat was calculated by adding the value of the score of each parameter studied.

\section{Production of AM enriched plasma}

Blood samples of 8 rats were taken in order to determine the baseline levels of AM [11]. One group $(n=4)$ was given $80 \mu \mathrm{mol}$ MFP/day orally for 30 days in order to obtain plasma with higher concentrations of AM than normal (AM-enriched plasma: AEP). Previous studies have shown that 30 days is enough time to produce increased levels of AM in plasma $[10,11]$. The other group received distilled water orally during the same period of time (normal AM plasma levels ANP). At the end of this period, blood was drawn by cardiac puncture and levels of AM in plasma were measured to confirm the effect of MFP (data not shown).

\section{AM purification from rat plasma}

Plasma from rats was chromatographed on a column of Sephadex $^{\circledR}$ G100 (Pharmacia Fine Chemical, Uppsala, Sweden). Fractions of $0.5 \mathrm{ml}$ were collected and AM content was analyzed by western blot [19] in order to select the fraction of the $S$ column containing AM.

\section{Statistical analysis}

Statistical analysis was performed with R 2.14.0 (R Development Core Team. 2011. R: A language and environment for statistical computing. R Foundation for Statistical Computing, Vienna, Austria). Differences were considered significant if $\mathrm{p}<0.05$.

\section{Results}

Amylase increased significantly $24 \mathrm{~h}$ after surgery in all groups ( $\bullet$ Table 1). However, the values did not correlate with score. As a consequence it has not been used for the classification in rats with and without pancreatitis. Histological evaluation showed that animals with pancreatitis treated with AM enriched plasma (AEP) or purified alpha-macroglobulin (PAM) had less damage to the pancreatic parenchyma compared to groups with pancreatitis receiving no treatment (C). A decrease of approximately 3 units in the score was observed in groups treated with AEP and PAM compared with animals without treatment ( $\bullet$ Table 2 ). In addition, the percentage or rats with pancreatitis was determined for each group. A value of the histological score higher than 4 and the death as a consequence of pancreatitis were considered to classify the animals as with pancreatitis. The value of 4 was selected considering the value of the mean of Sham group plus 2 standard deviations. An important decrease in the percentage of rats with pancreatitis was observed in animals

Table 1 Plasma levels of amylase (UI/ml). See material and methods for acronyms. Results are shown as mean \pm SEM.

\begin{tabular}{|lll} 
& Basal & $\mathbf{2 4 h}$ \\
\hline Sham & $22.9 \pm 2.8$ & $41.5 \pm 3.2^{*}$ \\
\hline C & $23.7 \pm 2.2$ & $91.8 \pm 36.2^{*}$ \\
\hline SS & $26.1 \pm 3.4$ & $61.1 \pm 16.1^{*}$ \\
\hline ANP & $30.4 \pm 3.9$ & $43.4 \pm 8.1^{*}$ \\
\hline AEP & $26.9 \pm 6.3$ & $42.0 \pm 8.1^{*}$ \\
\hline PAM & $29.1 \pm 4.0$ & $64.1 \pm 9.3^{*}$ \\
\hline
\end{tabular}

* indicates difference compared to basal value, $\mathrm{p}<0.05$ paired Student $T$ test

Table 2 Score of histological damage in experimental groups. See material and methods for acronyms. Results are shown as mean \pm SEM. ANOVA, $\mathrm{p}<0.05$.

$\begin{array}{ll}\text { Sham } & \mathbf{2 . 1 \pm 0 . 6} \\ \text { C } & 6.7 \pm 1.0^{b} \\ \text { SS } & 6.5 \pm 0.9^{b} \\ \text { ANP } & 5.9 \pm 1.3 \\ \text { AEP } & 3.4 \pm 0.6^{a} \\ \text { PAM } & 3.1 \pm 0.5^{a}\end{array}$

a indicates significant difference compared to $C$

bindicates significant difference compared to Sham, Tukey test 
Table 3 Number and percentage of rats with pancreatitis.

\begin{tabular}{|c|c|c|c|c|c|c|}
\hline & Sham & C & SS & ANP & AEP & PAM \\
\hline $\begin{array}{l}\text { number of rats with } \\
\text { pancreatitis }\end{array}$ & 4 & 14 & 12 & 12 & 4 & 5 \\
\hline $\begin{array}{l}\text { number of rats without } \\
\text { pancreatitis }\end{array}$ & 12 & 2 & 4 & 4 & 12 & 11 \\
\hline$\%$ of rats with pancreatitis & $25^{*}$ & 88 & 75 & 75 & $25^{*}$ & $31^{*}$ \\
\hline
\end{tabular}

Table 4 Plasma levels of alpha-macroglobulin. See material and methods for acronyms. Results are shown as mean \pm SEM. Repeated measures ANOVA, $p>0.05$.

\begin{tabular}{|lllll|} 
& \multicolumn{5}{c}{ alpha-macroglobulin $(\boldsymbol{\mu m o l} / \mathbf{l})$} \\
& $\mathbf{0 h s}$ & $\mathbf{2 4} \mathbf{h s}$ & $\mathbf{4 8} \mathbf{h s}$ & $\mathbf{7 2} \mathbf{h s}$ \\
\hline Sham & $21.8 \pm 3.7$ & $20.4 \pm 6.8$ & $22.6 \pm 4.4$ & $22.4 \pm 3.4$ \\
\hline C & $21.3 \pm 4.2$ & $18.2 \pm 4.3$ & $19.0 \pm 3.2$ & $19.6 \pm 4.9$ \\
\hline SS & $19.8 \pm 3.3$ & $23.0 \pm 4.0$ & $18.9 \pm 1.4$ & $22.1 \pm 3.3$ \\
\hline ANP & $19.8 \pm 3.2$ & $12.3 \pm 1.2$ & $15.1 \pm 2.3$ & $10.4 \pm 1.3$ \\
\hline AEP & $19.3 \pm 2.9$ & $13.0 \pm 2.9$ & $12.5 \pm 2.2$ & $17.1 \pm 4.2$ \\
\hline PAM & $21.6 \pm 1.0$ & $20.1 \pm 3.2$ & $21.9 \pm 3.4$ & $25.0 \pm 2.9$ \\
\hline
\end{tabular}

injected with AM (group PAM) and with AM enriched plasma (group AEP) ( $\triangle$ Table 3 ). The injection of saline solution or plasma with normal levels of AM did not change the percentage of rats with pancreatitis. It is important to consider that even among animals without ICDL (Sham group) there were cases in which some sort of damage to the pancreatic parenchyma was present ( $\bullet$ Table 2 ) and in which an increase in plasma amylase was observed.

Plasma concentration of AM showed no statistically significant differences among the experimental groups after $72 \mathrm{~h}$ ( $\odot$ Table 4 ), although a slightly increase was observed in PAM. Plasma levels of AM did not correlate with score.

\section{Discussion \\ $\nabla$}

Drug therapy of acute pancreatitis has not produced satisfactory results, and treatments meant to inhibit the self-digestive process have been ineffective mainly because the early pathophysiology of the disease is poorly understood. It has previously been shown that MFP administered before the surgical induction of pancreatitis is able to increase the survival of rats, attenuates the experimental course of acute pancreatitis, and reverses its histological signs. Morover, when animals receive MFP after the induction of pancreatitis, the pancreatic damage is lower. Previous studies from our laboratory showed that the administration of MFP disturbs the homeostasis of AM increasing its plasma levels $[10,11]$. The inhibitory effect of AM on proteinases suggests for it a protective role in the development of pancreatitis. The role of AM in the acute attack is undisputed and, once pancreatitis is established, its expression increases significantly [20]. These findings suggest a protective effect of MFP in the evolution of pancreatitis and that the increase of AM could be the limiting factor in the severity of acute pancreatitis. The experiments carried out in this study were performed to determine whether the administration of AM has a protective effect on the development of experimental pancreatitis.

The experiments showed that the score of pancreatic damage in rats that received plasma enriched with AM (AEP) and purified AM (PAM) was lower than in rats that did not receive it. This protective effect could be due to the AM injection. The effect of AEP and PAM can not be the consequence of MFP, because AEP has been obtained after $24 \mathrm{~h}$ of the last dose, when MFP has completely cleared from plasma. The fact that the SS group had the same level of damage and percentage of disease than controls rules out a possible beneficial effect of volume replacement on the progression of pancreatitis. The similarity of score values between groups C, ANP and SS, all with the greater pancreatic damage, further supports this conclusion. While previous results support the hypothesis that the administration of AM could be responsible for a less aggressive evolution of the disease, it is not possible to exclude the presence of other factors in the plasma enriched with AM as the cause of this effect.

The score of pancreatic damage and the percentage of animals with pancreatitis in the group treated with purified AM were similar to the sham group, a result that guarantees that AM may have an important role in the prevention of pancreatic damage.

\section{Conclusions}

The administration of autologous plasma enriched with AM or purified AM attenuates the development of pancreatic damage in rats with experimental pancreatitis. Thus AM could affect, in a beneficial manner, the evolution of acute pancreatitis and its systemic complications.

\section{Conflict of Interest Statement \\ $\nabla$}

The authors state no conflict of interest.

References

1 Saluja AK, Steer MLP. Patophysiology of pancreatitis. Role of citokines and other mediators of inflammation. Digestion 1999; 60: 27-33

2 Frossard JL, Hadengue A, Pastor CM. New serum markers for the detection of severe acute pancreatitis in humans. Am J Respir Crit Care Med 2001; 164: 162-170

3 Borth $W$. Alpha-2-macroglobulin, a multifunctional binding protein with targeting characteristics. FABEB J 1992; 6: 3345-3353

4 Sottrup-Jensen L. Alpha-macroglobulins: structure, shape and mechanism of proteinase complex formation. J Biol Chem 1989; 264: 11539-11542

5 Bísaro de Lorenc L, Ramos AM, Sánchez MC et al. Structural evaluation of plasma alpha2-macroglobulin in acute pancreatitis. Clin Chem Lab Med 2005; 43: 1183-1189

6 Seta T, Noguchi Y, Shimada $T$ et al. Treatment of acute pancreatitis with protease inhibitors: a meta-analysis. Eur J Gastroenterol Hepatol 2004; 16: 1287-1293

7 Demols A, Deviere J. New frontiers in the pharmacological prevention of post-ERCP pancreatitis: the cytokines. J Pancreas 2003; 4: 49-57

8 Reginster JY, Felsenberg D, Pavo I et al. Effect of raloxifene combined with monofluorophosphate as compared with monofluorophosphate alone in postmenopausal wonen with low bone mass: a randomized, controlled trial. Osteoporosis Int 2003; 14: 741-749

9 Rigalli A, Esteban L, Pera $L$ et al. Binding of monofluorophosphate to alpha-2-macroglobulin and C3. Calcif Tissue Int 1997; 60: 86-89

10 Di Loreto V, Rigalli A, Cinara L et al. Effect of disodium monofluorphosphate on plasma and blood viscosity in the rat. Clinic Hemorheol Microcirc 2008; 40: 259-265 
11 Di Loreto V, Roma SM, Menoyo I et al. Effect of the administration of monofluorophosphate on alpha-macroglobulin levels and clinical course of pancreatitis in rats. Arzneimittelforschung 2008; 58 : 136-140

12 Roma SM, Di Loreto V, Rigalli A. Effect of the treatment with monofluorophosphate on survival and pancreatic damage in rats with pancreatitis. Acta Gastroenterol Latinoam 2010; 40: 130-135

13 Guide to the care and use of experimental animal. Volume 1. Olfert ED, Cross BM, McWilliam AA (eds.). Canadian Council on animal care Guidelines. 1993

14 Sugimoto M, Takada T, Yasuda H. A new experimental pancreatitis by incomplete closed duodenal loop: the influence of pancreatic microcirculation on the development and progression of induced severe pancreatitis in rats. Pancreas 2004; 28: 112-119
15 Lupo $M$, Di Loreto $V$, Rigalli A. Substances administration. In: Experimental surgical models in the laboratory rat. Rigalli A, Di Loreto V. (eds.). Boca Ratón, FL: CRC Press, Taylor \& Francis Group, 2009; 55-62

16 Rigalli A. Euthanasia. In: Experimental surgical models in the laboratory rat. Rigalli A, Di Loreto V (eds.). Boca Ratón, FL: CRC Press, Taylor \& Francis Group, 2009; 31-32

17 Niederau C, Ferrel L, Grendell J. Caerulein-induced acute necrotizing pancreatitis in mice: protective effects of proglumide, benzotrip and secretin. Gastroenterology 1985; 88: 1192-1204

18 Klöppel G. Acute pancreatitis. Semin Diagn Pathol 2004; 21: 221-226

19 Laemmli VK. Cleavage of structural proteins during the assembly of the head of bacteriophage T4. Nature 1970; 227: 680-685

20 Fétaud V, Frossard JL, Farina A et al. Proteomic profiling in an animal model of acute pancreatitis. Proteomics 2008; 8: 3621-3631 